\title{
Surface-Based Structural Group Analysis of fMRI Data
}

\author{
Grégory Operto ${ }^{1}$, Cédric Clouchoux ${ }^{1}$, Rémy Bulot ${ }^{1}$, Jean-Luc Anton ${ }^{2}$, \\ and Olivier Coulon ${ }^{1}$ \\ ${ }^{1}$ Laboratoire LSIS, UMR CNRS 6168, Marseille, France \\ gregory.operto@univmed.fr \\ ${ }^{2}$ Centre d'IRM fonctionnelle de Marseille, Marseille, France
}

\begin{abstract}
As structural and surface-based analyses gain interest for activation detection, morphometry and intersubject matching purposes, this paper proposes a method to perform structural group analyses directly on the cortical surface. Scale-space blobs are extracted from surface-based functional maps and matched across subjects. The process aims at identifying activations within a population despite the various effects due to variability. Results of the method are presented with simulated activations and with data from a somatotopy protocol.
\end{abstract}

\section{Introduction}

Group analysis in fMRI aims at building descriptions of unicity and diversity across a pool of subjects of a same cognitive experiment. When the pool is suficiently big, results may then be generalized to a population. Still, matching these subjects together strongly suffers from the intersubject variability that exists at different levels : anatomical, physiological, functional [1. The usual procedure consists in normalizing every scanned subject to a common anatomical space. Hence, any location in that space is supposed to correspond to the same region in the brain of each subject. Since responses to a same protocol show variability from one subject to another, decisions concerning a group-scale effect are taken upon the hypothesis that the signals across subjects are normally distributed. This forms the general basis of any voxel-based activation detection technique. This general, historical and widely spread volume-based approach consider the voxels of the whole brain, including those in the white matter, whereas the main sources of the functional signal are located in the cortical ribbon. In this context, surface-based analysis schemes especially gain interest [2/3/4 as they focus on the main location of the cerebral activity. On the other hand, the framework of structural approaches [5]6] allows to match objects from one subject to another instead of voxels, a level at which variability is better addressed. In this paper, the proposed method aims at performing analyses of functional data across a group of subjects, in both a surface-based and a structural way (as opposed to the iconic voxel-based fashion). This approach gains sensitivity against volumebased approaches, first by restricting the analysis to the cortex and by focusing

D. Metaxas et al. (Eds.): MICCAI 2008, Part I, LNCS 5241, pp. 959-966, 2008.

(C) Springer-Verlag Berlin Heidelberg 2008 
on objects rather than voxels. The method is inspired by the volume-based structural group analysis presented in [5]. After adapting its general algorithms to surfaces, such as building surface-based scale-space primal sketches, the intersubject anatomical matching is addressed via a 2D coordinate system defined on the cortical surface [2]. In this context, this work attempts to gather the advantages of two approaches, structural and surface-based, in order to overcome the intersubject variability as much as possible. This paper details in section 2 the method from the representation of data to the detection itself. Section 3 presents results on synthetic and real data. Discussion is then presented in section 4 .

\section{Surface-Based Structural Group Analysis}

\subsection{Data Preparation}

For each subject, a triangulated mesh of the inner cortical surface $(\mathrm{G} / \mathrm{W}$ interface) is first extracted from their anatomical volume using the Brainvisa package 7]. The advantages of using this surface reside in its geometry and its spherical topology. Functional volumes are coregistered with the anatomy, e.g. using SPM2 package [8]. Surface-based representations of functional data are then created using a volume-to-surface projection technique proposed in [9] and tmaps are built by an incremental statistical method [10. Cortical localization and intersubject matching are performed via a surface-based coordinate system built on each cortical surface 2. This pipeline finally allows to build individual surface-based statistical maps, used as inputs for the structural representation process described below.

\subsection{Structural Representation of Data}

The structural approach in fMRI data analysis is advocated by different works [56] for theoretical, computational and representation purposes. The voxels are only the acquisition space and have never had any anatomical meaning, other than the simple localization provided by spatial normalization. Moreover, the big amounts of information contained in raw 3D volumes lead to high computation costs, and not necessarily higher sensitivity. The main idea of the structural approach is to deal with representations of data closer to the objects under study than voxels. Some existing works choose to build a scale-space primal sketch of the activity, considering that objects of interest are found at multiple scales 11,12. This multiscale approach is advocated for instance by [5]13. Some others focus on supra-threshold regions [14, or activity peaks [1. Subsequent objects are hence characterized by different, numerical or geometrical, features.

One part of the work presented here consisted in adapting the volume-based scale-space primal-sketch construction algorithm to a topologically and geometrically different domain such as a triangulated surface. Linear scale-space representations of the surface-based functional maps are obtained by smoothing along the meshes by solving the heat-equation, leading to a diffusion process which progressively removes details as in figure 1. In order to solve the heat 
equation on a triangular mesh, the laplacian is computed using a finite element approximation [15].

The method then defines a hierarchical description of the structure of each statictical map on the cortical surface. Different characteristics can be embedded with the scale-space blobs, from their lifetime across scales to geometrical or intensity-based measurements. We chose to attach the product between the blob's lifetime and the t-test value of the blob's maximum on the original functional map, hence describing both the intensity of the blobs and their saliency with respect with surrounding structures [513]. This has been shown to be an appropriate measurement for activation detection purpose [5].
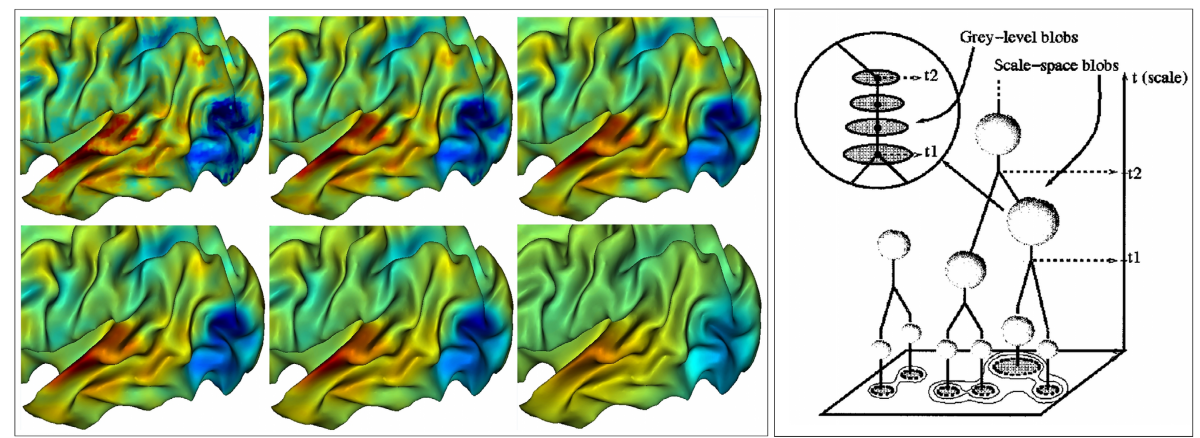

Fig. 1. (left) Example of scales from a scale-space of a surface-based statistical t-map (right) Symbolic representation of a scale-space primal sketch

\subsection{Structural Analysis on the Cortical Surface}

Let $n_{s}$ be the number of subjects responding to a certain cognitive task. We build for each one a surface-based t-map, describing the relevance for each node to have been activated by the task. $n_{s}$ primal sketches are created from these t-maps, with $n_{b i}$ the number of scale-space blobs extracted from t-map $i, i \in\left\{1, \ldots, n_{s}\right\}$. By matching the primal sketches, the structural analysis aims at assigning a specific label to each of the $N=\sum_{i \in\left[1, \ldots, n_{s}\right]} n_{b_{i}}$ scale-space blobs, depending on the blob probability to represent an actual activation. As far as the matching can be achieved, corresponding activation clusters get assigned the same positive label across subjects and null label is given to blobs of non-interest (noise). This association relies on a model defined using the same set of rules as in [5], i.e. :

1. A blob representing an activation is likely to have high measurements;

2. Two blobs representing the same activation must be linked in the graph and have the same non-null label;

3. Two blobs representing the same activation (same non-null label) are likely to have spatial supports close to each other;

4. An activation is represented only once for each subject, ie, a positive label must have only one occurrence per primal sketch. 
Group analysis is performed on a graph embedding primal sketches of all subjects and built as follow : graph nodes are blobs and edges are set between two blobs if those two blobs a) belong to two different subjects and b) are close enough in the common space defined by the surface-based coordinate system defined in [2] (namely, inter-blob distance must be below $20 \mathrm{~mm}$ ).

As presented in [5], a blob is identified as an activation if its associated measurements are sufficiently high in the map and if it is repeated often enough across subjects. The label field is modeled as a Markov random field (MRF), whose optimal realization can be achieved in a Bayesian framework by maximizing the posterior probability $P(X \mid Y)$, with $X$ the label field on the primal sketch graph and $Y$ the data. In other terms, maximization of this probability drives to the optimal labeling given the characteristics of the blobs (measurements, scale) and the similarities observed between subjects. It is shown 5 to be equivalent to the minimization of an energy function $U(X \mid Y)$ defined as follow :

$$
U(X \mid Y)=\sum_{s=1}^{N} V\left(y_{s} \mid x_{s}\right)+\sum_{c \in C} V_{c}\left(\left.X\right|_{c}\right)
$$

where $V\left(y_{s} \mid x_{s}\right)$ is a data-driven potential function (rule 1) and $V_{c}\left(\left.X\right|_{c}\right)$ a contextual potential function calculated from every contextual clique $c \in C$ of the graph (rule 2, 3 and 4). Chosen potential functions are the same as in [5] except for the one (figure 2) attached to second-order cliques, which deals with intersubject similarities. Similarity between two blobs is computed using an intersubject

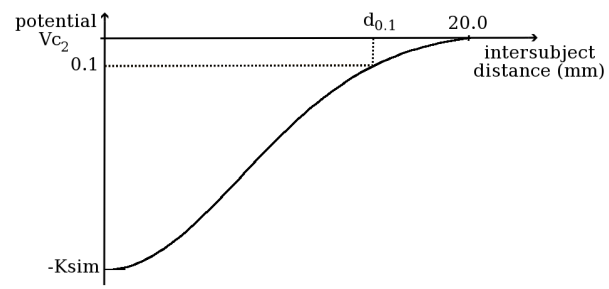

Fig. 2. The second-order inter-primal-sketches clique potential function

distance function $f\left(b_{1}, b_{2}\right)$. If $b_{1}$ and $b_{2}$ belong to different subjects and have the same non-null label, the potential $V_{c_{2}}$ then equals :

$$
V_{c_{2}}=-K_{s i m} \cdot \frac{e^{-f\left(b_{1}, b_{2}\right)^{2}}}{2 \cdot\left(\frac{d_{0.1}}{\sqrt{2 \cdot \log (10.0)}}\right)^{2}}
$$

Otherwise (different or null labels), $V_{c_{2}}=0$. The similarity function $f\left(b_{1}, b_{2}\right)$ is computed using the surface-based referential attached to each cortical mesh as a common referential. Given two blobs $b_{1}$ and $b_{2}, f\left(b_{1}, b_{2}\right)$ returns the length of the shortest geodesic path between the two blobs' maxima in the common referential. $d_{0.1}$ is a user-defined distance at which the potential $V_{c_{2}}$ equals 0.1 . 
Once all potential functions are defined, the total energy function is defined. To minimize it, we use a stochastic algorithm, the Gibbs sampler with annealing [16. After minimization, the process returns a set of positive labels, each one representing an activation and having an occurrence in a number of primal sketches. We therefore know the occurrence, or the non-occurrence, of each activation for any subject. These occurrences can then be mapped on the individual anatomy of the subject for localization considerations.

\section{Experiments}

\subsection{Experiments on Simulated Data}

The whole pipeline was applied to simulated activation maps on a spherical mesh. Gaussian background noise was first distributed all over the surface, then a set of 4 simulated activation clusters were added to the background. Since the experiment is run on a common spherical mesh, the similarity issue has no more anatomical meaning compared to as on real meshes. Still, the profile of the map was meant to recall the one of a typical functional t-map and some amount of variability was simulated in terms of activation intensity and location as illustrated on figure 3 : from the original location, a different node is randomly selected in a neighborhood as a new activation focus for each map. The neighborhood is taken as $10.0 \mathrm{~mm}$-wide, equal to the typical intersubject variability magnitude $\delta_{\tau}$ in [6]. Primal sketches are built for each of the 10 maps and minimization is run on the resulting graph and label field. Non-null labels are presented in figure 3. A set of 4 non-null distinct labels were attributed, each of them identifying the same specific simulated activation focus across all the maps. Blobs are represented as small spheres for visualization purposes.

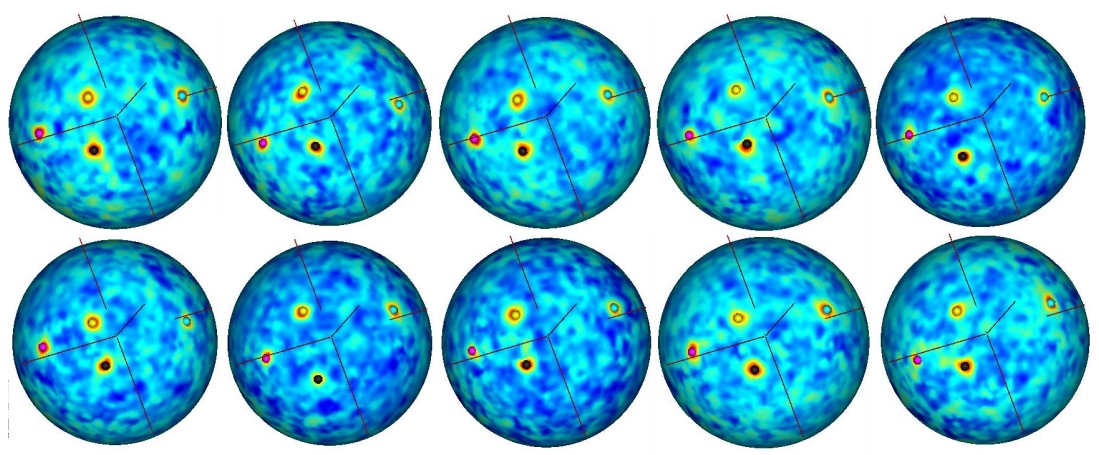

Fig. 3. Labeling after minimization on simulated activation maps and spherical atlas

\subsection{Experiments on Real Data}

We ran the activation detection pipeline on 8 subjects issued from a somatotopy experiment. Surface-based maps were computed for a simple (foot movementrest) contrast and primal sketches were extracted from these. Minimization was 
performed on the primal sketches graph. Resultingly, there were 9 non-null labels generated. One of them is repeated across the 8 subjects, two of them exist in 7 subjects and one can be found in 6 subjects. The most frequently occurring labels are gathered at the top of the central sulcus, or on the medial face of the hemisphere, located either in the primary sensorimotor areas (M1-S1) or in the supplementary motor area (SMA), as the results one would expect from a neuroscientific point of view. One label appears near the insular cortex in 2 subjects. Figure 4 shows non-null labels for 4 subjects, together with individual statistical t-maps thresholded at $p<0.05$ (corrected for multiple comparisons). Table 1 details the numbers of occurrences and the energies associated to these labels.

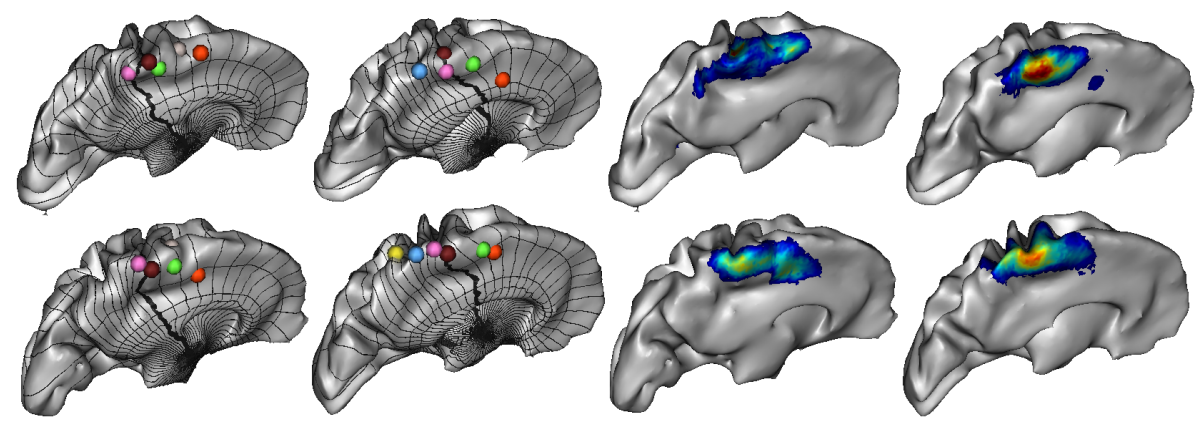

Fig. 4. Resulting activations from a right-motor contrast for 4 subjects. (left) labeled after minimization (right) thresholded individual statistical t-maps $(p<0.05$ corrected).

Table 1. Energies and numbers of occurences of non-null generated labels

\begin{tabular}{|c|c|c|c||c|c|c|c|}
\hline Label & Color & Global energy & Occurences & Label & Color & Global energy & Occurences \\
\hline 3 & 0 & -586.82 & 8 & 7 & 0 & -81.11 & 7 \\
\hline 5 & 0 & -368.92 & 6 & 10 & 0 & -42.91 & 2 \\
\hline 9 & 0 & -236.56 & 7 & 8 & 0 & -25.66 & 2 \\
\hline 11 & 0 & -134.71 & 3 & 12 & & -24.83 & 2 \\
\hline 4 & & -89.75 & 3 & & & & \\
\hline
\end{tabular}

\section{Discussion}

The minimization aims at finding the label configuration which leads to fit with the initial model. Results on simulated data show that non-null labels were attributed to areas on the sphere showing relative high intensity and good matching across the simulated maps. Specifically, all clusters were properly labelled, with no false positive. On real data, results show that non-null labels appear in variable numbers of subjects, which correlates with the variability effects recognized by other works e.g. [516]. Overall, the label with the lowest energy (table 1) is 
the one existing in most subjects and identifies the primary motor activation. Then, the labels with the second lowest energies stand for somatosensory activations on one hand, and supplementary motor activations on the other. This complies precisely with our model and with the neuroscientific results expected for such study. Figure 4 compares the activations to thresholded individual statistical maps and shows that several components may exist under a single suprathreshold cluster, which is a specific valuable feature of structural approaches.

In order to assess similarities between subjects, a surface-based coordinate system is used as a common referential to all subjects. However, these coordinates cannot be used as is to compute distances. This illustrates the need for a common reference spatial support, i.e. a cortical surface atlas. Without such an atlas, our method chooses one subject as a reference used to calculate interblobs distances. Nevertheless, this subject presents specific anatomical features, which are not necessarily representative neither at a global nor at the group scale. This inevitably introduces a bias in the calculated distances [17. The choice of a statistically representative and non-biased atlas is directly related to the performance in overcoming intersubject anatomical variability and therefore a crucial point in order to realize our measures on the cortical surface. By these considerations, structural group analysis using surface-based intersubject distances constitute a new approach, significantly different compared to voxel/node-based statistical methods.

\section{Conclusion and Further Work}

This paper presents a method which connects two distinct analysis frameworks, structural and surface-based. The method was applied to a set of simulated data and functional data from a somatotopy experiment. The results showed efficiency of the chosen model in retrieving simulated activations and detecting activations associated to a simple motor task. The paper underlines the importance of a common space to make comparisons between subjects. Future research will concern the creation of a cortical surface reference atlas and therefore the implementation of an anatomically meaningful intersubject distance.

\section{References}

1. Thirion, B., Flandin, G., Pinel, P., Roche, A., Ciuciu, P., Poline, J.B.: Dealing with the shortcomings of spatial normalization: Multi-subject parcellation of fMRI datasets. Human Brain Mapping 27(8), 678-693 (2005)

2. Clouchoux, C., Coulon, O., Riviere, D., Cachia, A., Mangin, J.F., Régis, J.: Anatomically constrained surface parameterization for cortical localization. In: Duncan, J.S., Gerig, G. (eds.) MICCAI 2005. LNCS, vol. 3750, pp. 344-351. Springer, Heidelberg (2005)

3. Andrade, A., Kherif, F., Mangin, J., Worsley, K., Paradis, A., Simon, O., Dehaene, S., Bihan, D.L., Poline, J.: Detection of fmri activation using cortical surface mapping. Human Brain Mapping 12, 79-93 (2001) 
4. Van Essen, D.C., Drury, H.A.: Structural and functional analyses of human cerebral cortex using a surface-based atlas. J. Neurosci. 17(18), 7079-7102 (1997)

5. Coulon, O., Mangin, J.F., Poline, J.B., Zilbovicius, M., Roumenov, D., Samson, Y., Frouin, V., Bloch, I.: Structural group analysis of functional activation maps. NeuroImage 11(6), 767-782 (2001)

6. Thirion, B., Pinel, P., Tucholka, A., Roche, A., Ciuciu, P., Mangin, J.F., Poline, J.B.: Structural analysis of fmri data revisited: Improving the sensitivity and reliability of fmri group studies. IEEE Transactions on Medical Imaging 26(9), 12561269 (2007)

7. http://brainvisa.info

8. Ashburner, J., Friston, K.: Voxel-based morphometry: the methods. Neuroimage $11,805-821(2000)$

9. Operto, G., Bulot, R., Anton, J.L., Coulon, O.: Projection of fmri data onto the cortical surface using anatomically-informed convolution kernels. Neuroimage 39(1), 127-135 (2007)

10. Roche, A., Lahaye, P.J., Poline, J.B.: Incremental activation detection in fMRI series using kalman filtering. In: IEEE International Symposium on Biomedical Imaging, pp. 376-379 (2004)

11. Worsley, K., Marrett, S., Neelin, P., Evans, A.: Searching scale space for activation in PET images. Human Brain Mapping 4, 74-90 (1996)

12. Poline, J.B., Mazoyer, B.: Analysis of individual brain activation maps using hierarchical description and multiscale detection. IEEE Transactions on Medical Imaging 13(4), 702-710 (1994)

13. Lindeberg, T., Lidberg, P., Roland, P.: Analysis of brain activation patterns using a 3-D scale-space primal sketch. Human Brain Mapping 7, 166-194 (1999)

14. Simon, O., Kherif, F., Flandin, G., Poline, J.B., Riviere, D., Mangin, J.F., Bihan, D.L., Dehaene, S.: Automatized clustering and functional geometry of human parietofrontal networks for language, space, and number. Neuroimage 23(3), 1192-1202 (2004)

15. Chung, M.K., Taylor, J.: Diffusion smoothing on brain surface via finite element method. In: IEEE International Symposium on Biomedical Imaging, vol. 1, pp. 432-435 (2004)

16. Geman, S., Geman, D.: Stochastic relaxation, gibbs distribution, and the bayesian restoration of images. IEEE Trans. on Pattern Anal. Machine Intell. 6, 721-741 (1984)

17. Devlin, J., Poldrack, R.: In praise of tedious anatomy. Neuroimage 37(4), 1033-1041 (2007) 